\title{
Kosten- und Risikostrukturen wichtiger Vertragsformen in der Maintenance ziviler Flugtriebwerke
}

\author{
Mike Steglich
}

\section{Problemstellung}

Die Maintenance ziviler Flugtriebwerke beinhaltet im Wesentlichen die Überholung bzw. die Reparatur von Flugtriebwerken von Luftfahrtgesellschaften oder sonstigen Betreibern durch einen Maintenance-Dienstleister. Da einerseits die Maintenance-Kosten einen erheblichen Anteil an den operativen Kosten des Betriebs eines Flugzeuges darstellen ${ }^{1}$, andererseits eine angespannte Konkurrenzsituation zwischen den einzelnen MaintenanceDienstleistern besteht ${ }^{2}$, besitzt die spezifische Ausgestaltung der Maintenance-Verträge zwischen den Betreibern der Flugtriebwerke (Operator) und den Maintenance-Dienstleistern (MRO-Shop ${ }^{3}$ ) eine sehr große Bedeutung fuir die Ergebnissituation der beiden Vertragspartner.

In diesem Aufsatz werden die wichtigsten Vertragsformen vorgestellt und hinsichtlich ihrer Kosten- und Risikostrukturen diskutiert. Aus der Kombination dieser Strukturen mit den grundsätzlichen Einstellungen entsprechender Entscheidungsträger gegenüber unsicheren Ergebnissen lassen sich Rangfolgen der Vorteilhaftigkeit der Vertragsformen erarbeiten, die abschließend in einem Modell zur Bestimmung der für beide Vertragspartner optimalen Vertragsform zusammengefasst werden.

\section{Die Kostenstrukturen der Maintenance von Flugtriebwerken}

Aufgrund des elementaren Einflusses der Instandsetzungskosten der Flugtriebwerke auf die Ergebnissituation beider Vertragspartner besitzen die Kenntnis und Steuerung derartiger Maintenance-Kosten eine große Bedeutung für den Triebwerkseigner und den MROShop. Grundsätzlich werden die Maintenance-Kosten von der Art der Instandhaltungs-Ereignisse (Shop Visits) und der Kostenstruktur dieser Shop Visits bestimmt.

\subsection{Arten von Shop Visits}

Da Flugtriebwerke vielfältigen Sicherheitsaspekten unterliegen, werden sie in definierten Intervallen einer teilweisen oder vollständigen Überholung unterzogen. Hinsichtlich der Steuerung dieser geplanten Überholungsereignisse existieren zwei grundsätzliche Vorgehensweisen. Gemäß der Hard Time Method finden die Überholungsereignisse nach einer festgelegten Anzahl von Flugstunden statt. Im Gegensatz zu dieser Vorgehensweise werden die Überholungen gemäß der On Condition Method entsprechend dem Zustand der ein- zelnen Triebwerke durchgefuihrt. Zusätzliche Faktoren der Steuerung der Überholungsereignisse stellen lebenszeitbegrenzte Teile (Life Limited Parts $=$ LLP) dar. ${ }^{4}$ Für derartige Teile sind Grenzwerte definiert, die im Einsatz nicht überschritten werden dürfen und spätestens mit dem Erreichen des entsprechenden Wertes zu einem Shop Visit führen. In Abhängigkeit von der (vom Triebwerksbetreiber beeinflussbaren) Abnutzung der Triebwerke und den zu beachtenden Grenzwerten für die LLPs können die einzelnen Überholungsereignisse hinsichtlich der Kosten und der Belange des reibungslosen Flugbetriebs der entsprechenden Fluggesellschaft optimal geplant werden. Betrachtet man für ein einzelnes Triebwerk einen entsprechend langen Zeitraum, ergibt sich für dieses Triebwerk eine Abfolge von geplanten Überholungsereignissen, wobei die einzelnen aufeinanderfolgenden Überholungen einen unterschiedlichen Bearbeitungsumfang (Workscope) aufweisen können.

Zusätzlich zu diesen geplanten Ereignissen können u. a. aufgrund externer Einfluisse (z. B. Blitz- oder Vogelschlag) oder interner Ereignisse (z. B. Materialfehler) Zustände an dem jeweiligen Triebwerk auftreten, die letztlich zu einem ungeplanten Shop Visit fuihren. Der Eintritt derartiger Ereignisse kann nicht exakt geplant, aber anhand empirischer Daten der betrachteten Flotte oder anderer veröffentlichter Schadensdaten prognostiziert werden. Die Informationen über die geplanten Überholungsereignisse und die prognostizierten ungeplanten Ereignisse werden einschließlich der unterschiedlichen Workscopes in einem Shop Visit Forecast zusammengefasst.

\subsection{Der Instandsetzungsprozess}

Die einzelnen geplanten und ungeplanten Shop Visits unterliegen im MRO-Shop einem grundsätzlichen Arbeitsablauf. Dieser allgemeine Instandsetzungsprozess und die einzelnen Workscopes bestimmen die Struktur und die Ausprägung der Kosten eines MRO-Shop Visits. Wie in Abbildung 1 vereinfacht dargestellt, beginnt jede Triebwerksinstandsetzung nach Abschluss des Wareneingangs mit der Demontage des Triebwerks in einzelne Module bzw. bis auf Einzelteiltiefe. Diese Einzelteile werden gereinigt und in einem nächsten Schritt einer Untersuchung auf mögliche Schadensfälle unterzogen. Insofern Triebwerksteile ohne Schadensbefund sind, können sie wieder im Triebwerk verbaut werden. Liegt ein Schadensfall vor, ist anhand spezifischer Vorgaben des Originalherstellers bzw. der Luftfahrtbehörden zu entscheiden, ob die entsprechenden Teile zu verschrotten oder reparaturfähig sind. Schrottteile sind durch Neuteile oder flugfähige Gebrauchtteile zu ersetzen. Reparaturfähige Teile werden 


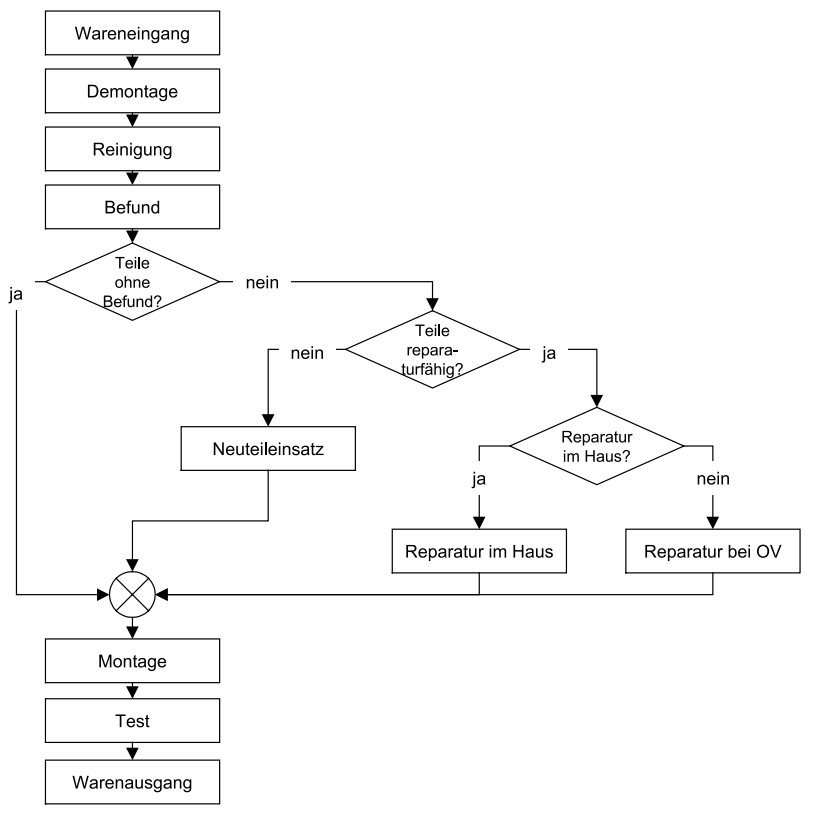

Abb. 1: Instandhaltungsprozess

gemäß den Reparaturfähigkeiten und den Kapazitäten des MRO-Shops entweder im Haus oder bei Reparaturanbietern (Outside Vendor - OV) repariert. Wenn alle wieder verwendbaren Teile, alle neuen bzw. gebrauchten Austauschteile und die reparierten Teile (oder ggf. adäquate Austauschteile) vorliegen, kann die Montage der einzelnen Triebwerksmodule und letztlich des Triebwerks erfolgen. Vor dem Warenausgang und der Auslieferung an den Kunden ist das Triebwerk einem Testlauf zu unterziehen, um so die geforderten Parameter und die Flugfähigkeit nachzuweisen.

\subsection{Die Kostenstruktur eines Shop Visits}

Die beschriebenen Arbeitsabläufe des Instandsetzungsprozesses führen zu spezifischen Kosten, die sich in die Eigenleistungskosten (Labour), die Materialkosten, die Kosten für Fremdleistungen (Outside Vendor) und die sonstigen Kosten gliedern lassen. ${ }^{5}$

Den größten Kostenblock bilden in der Regel die Materialkosten der Neu- oder Gebrauchtteile, die als Austauschteile fuir die zu verschrottenden Teile in das Triebwerk eingehen. Weitere Materialkosten werden durch den Ersatz von Verbrauchs- und Verschleißteilen, durch den Bedarf an Hilfs- und Betriebsstoffen sowie durch den Materialeinsatz der im Haus ausgeführten Reparaturen verursacht. Kosten für Fremdreparaturen fallen für die von einem Outside Vendor reparierten Triebwerksteile in Höhe der Rechnung dieses Anbieters an.

Die Eigenleistungskosten repräsentieren die Arbeitsleistung der direkt am Triebwerk tätigen Mitarbeiter in Arbeitsstunden und der verursachten Fertigungseinzelkosten. ${ }^{6}$ In der Regel werden die Eigenleistungen für Reparaturen und für die Basic Labour getrennt ausgewiesen, wobei die Basic Labour die Arbeitsleistungen aller nicht der Reparatur zugehörigen Arbeitsschritte des Instandsetzungsprozesses (Wareneingang, Demontage, Reinigung, Befund, Montage, Test und Warenausgang) abbildet.
Unter den sonstigen Kosten werden im Wesentlichen Sondereinzelkosten des Vertriebs, wie z. B. Transportkosten, Kosten für Leihtriebwerke, Provisionen etc., und weitere dem Shop Visit direkt zurechenbare Kosten zusammengefasst.

Zusätzlich zu diesen Kosten, die einem Shop Visit direkt zugerechnet werden können, sind die gemäß dem Beanspruchungs- und ggf. dem Einwirkungsprinzip zurechenbaren Kosten in die Kalkulation eines Shop Visits einzubeziehen. ${ }^{7}$ Grundsätzlich sind diesem Kostenblock die Kosten für Kapazitäten der Betriebsbereitschaft zuzuordnen, die von einem betrachteten Shop Visit beansprucht werden, wie z. B. die bewerteten Personalkapazitäten fuir Triebwerksingenieure, Testingenieure sowie sonstiges indirektes Personal, die Kosten für Anlagekapazitäten und die Kosten der zur Montage bzw. Demontage eines Triebwerks benötigten Betriebsmittel und Werkzeuge.

\section{Wichtige Vertragsformen}

Hinsichtlich der vertraglichen Gestaltung der Geschäftbeziehungen zwischen dem Operator und dem MROShop existiert eine Vielzahl von Möglichkeiten, wobei sich am Markt die Time and Material-Verträge (T\&M), die Fly by Hour-Verträge $(\mathrm{FbH})$ und die Verträge mit einer Garantie maximaler Maintenance-Kosten (Garanteed Maintenance Costs - GMC) als wichtigste Vertragsformen durchgesetzt haben. ${ }^{8}$

\subsection{Merkmale von Verträgen}

Zur Beschreibung der Eigenschaften dieser Vertragsformen existieren mit der Exklusivität der Leistungsvergabe und des Abrechnungsmodus zwei Merkmale, die im Folgenden kurz vorgestellt werden.

Im Sinne der Exklusivität der Leistungsvergabe können exklusive und nichtexklusive Verträge unterschieden werden. Im Fall exklusiver Verträge ist der Operator mit dem im Vertrag definierten Leistungsumfang an den MRO-Shop gebunden. Alle im Leistungsumfang enthaltenen Shop Visits sind vom Operator an den MRO-Shop zu vergeben. Derartige Verträge besitzen in der Regel eine langfristige Vertragslaufzeit von 5-10 Jahren. Ist der Operator nicht exklusiv an einen MRO-Shop gebunden, kann er für jedes einzelne geplante oder ungeplante Instandsetzungsereignis entscheiden, welcher MROShop mit der Ausfuihrung des entsprechenden Shop Visits beauftragt wird. Damit gelten diese Verträge grundsätzlich nur für den oder die einzeln beauftragten Shop Visits.

Als Abrechnungsmodi existieren Einzelabrechnungen, Festpreise und Raten je Flugstunde. Bei Einzelabrechnungen werden alle Bestandteile eines Shop Visits anhand der verhandelten Konditionen separat abgerechnet. Mit dem Ausmaß des jeweiligen Workscopes variiert der vom Operator zu zahlende Preis. Im Gegensatz dazu wird bei einem Festpreis für eine definierte Leistung ein 
fester vom Operator zu zahlender Preis vereinbart. Festpreisvarianten können auch in einer definierten Höchstgrenze der vom Operator an den Shop zu leistenden Zahlungen bestehen. Bei einer Abrechnung mit einer Rate je Flugstunde hat der Operator einen bestimmten im Vertrag definierten Geldbetrag je geflogener Stunde an den MRO-Shop zu zahlen, mit dem ein definiertes Leistungsspektrum in einem bestimmten Zeitraum abgedeckt wird.

\subsection{Time and Material-Verträge}

Bei T\&M-Verträgen handelt es sich um Verträge mit Einzelabrechnung aller Bestandteile eines Shop Visits, fuir die in der Regel keine exklusive Bindung des Kunden an den MRO-Shop existiert. ${ }^{9}$ Für die Erörterung dieser Vertragsform sei auf die vereinfachte Kosten- bzw. Erlöskalkulation eines Shop Visits (mit fiktiven Werten) aus der Sicht des MRO-Shops in Tabelle 1 verwiesen.

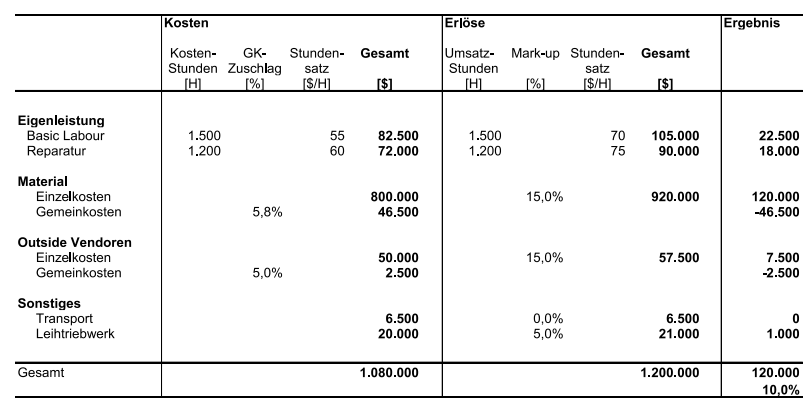

Tab. 1: T\&M-Kalkulation mit fiktiven Werten

Bei der Selbstkostenkalkulation handelt es sich um eine differenzierte Zuschlagskalkulation. Die Eigenleistungskosten ergeben sich aus der Multiplikation der geleisteten Stunden mit den entsprechenden Kostenstundensätzen. Diese Stundensätze bilden die Fertigungseinzelkosten und die gemäß dem Beanspruchungsprinzip zurechenbaren Kapazitätskosten der jeweiligen Unternehmensbereiche ab. Für Material und beauftragte Reparaturen fallen Einzelkosten an, denen Gemeinkosten beanspruchungsgerecht zugerechnet werden. Unter Sonstiges werden in diesem Beispiel Kosten für Transport und fuir die Bereitstellung eines Leihtriebwerks zusammengefasst.

Die Erlöskalkulation bildet die Grundlage der Rechnungslegung gegenuiber dem Operator, wobei alle Kostenbestandteile des Shop Visits entsprechend den vereinbarten Konditionen separat abzurechnen sind. Die Eigenleistung wird dem Operator anhand der geleisteten Arbeitsstunden in Rechnung gestellt, die mit den vereinbarten Erlösstundensätzen multipliziert werden. Die Abrechnung für Neumaterial erfolgt gemäß den Verbrauchsmengen der einzelnen im Triebwerk ausgetauschten Teile, die mit den aktuellen Listenpreisen der Originalhersteller multipliziert werden. Auf diesen Wert wird abschließend ein im Vertrag vereinbarter prozentualer Aufschlag auf den Listenpreis (Mark-Up) aufgeschlagen. Gebrauchtes Material wird analog abgerechnet, wobei statt des Listenpreises ein marktuiblicher Wert für die entsprechenden Teile angesetzt wird. Die Rechnungen der mit den Reparaturen beauftragten Repara-
tur-Shops werden, mit einem Aufschlag versehen, dem Operator weiter berechnet. Die Abrechnung der sonstigen Kosten erfolgt analog zu den beschriebenen Material- bzw. Outside Vendor-Abrechnungen.

Das Beispiel wird gemäß Tabelle 2 um die Annahme erweitert, dass der Operator den MRO-Shop über eine Dauer von fünf Jahren mit den anfallenden Shop Visits einzeln beauftragt. Verknüpft man die in Tabelle 1 gegebenen Kosten und Erlöse je Triebwerk mit der Anzahl der Shop Visits in den einzelnen Jahren, erhält man die Erlöse, Kosten und letztlich die Ergebnisse in den einzelnen Jahren aus der Sicht des MRO-Shops. In diesem Zusammenhang wird vereinfachend unterstellt, dass keine Preissteigerungseffekte fuir die einzelnen Kostenbestandteile auftreten. Insofern sich der MRO-Shop einzig an Gewinngrößen orientiert, kann man über die Summe der jährlichen Ergebnisse den Erfolg eines solchen Vertrags (im Beispiel 720 T \$) ermitteln. ${ }^{10}$ Aus Sicht des Operators entsprechen die Kosten den Erlösen des MRO-Shops.

\begin{tabular}{|c|c|c|c|c|c|c|c|}
\hline & & 2003 & 2004 & 2005 & 2006 & 2007 & Gesamt \\
\hline Anzahl Shop Visits & & 0 & 1 & 2 & 2 & 1 & 6 \\
\hline \multicolumn{8}{|l|}{ MRO-Shop } \\
\hline Erlöse & {$[T \$]$} & 0 & 1.200 & 2.400 & 2.400 & 1.200 & 7.200 \\
\hline Kosten & {$[T \$]$} & 0 & 1.080 & 2.160 & 2.160 & 1.080 & 6.480 \\
\hline Ergebnis & [T\$] & 0 & 120 & 240 & 240 & 120 & 720 \\
\hline \multicolumn{8}{|l|}{ Operator } \\
\hline Kosten & [T \$] & 0 & 1.200 & 2.400 & 2.400 & 1.200 & 7.200 \\
\hline
\end{tabular}

Tab. 2: Mehrperiodige Ergebnisse eines T\&M-Vertrages mit fiktiven Werten

Für T\&M-Verträge existieren eine Anzahl von Variationen. So können Teile der T\&M-Rechnungen (z. B. die Arbeitsleistungen für die Basic Labour) mit einem Festpreis verbunden sein. Eine weitere Festpreisvariation besteht in einem so genannten Not To Exeed Price (NTEP), der eine nicht zu überschreitende Obergrenze der Time and Material-Abrechnung definiert. ${ }^{11}$ Oft werden NTEPAbrechnungen mit einem exklusiven Vertrag verbunden.

\subsection{Fly by Hour-Verträge}

FbH-Verträge lassen sich als exklusive Verträge mit einer Abrechnung auf der Basis einer Rate pro Flugstunde klassifizieren. Der Kunde zahlt eine vereinbarte Rate pro Flugstunde, mit der die Kosten der vertraglich einzubeziehenden Shop Visits zuzüglich eines Ergebnisbeitrags für den MRO-Shop abgedeckt sind. ${ }^{12}$ Zur Berechnung der FbH-Rate sind in einem ersten Schritt die Maintenance-Kosten entsprechend dem vereinbarten Leistungsspektrum und den betroffenen Triebwerken für die gesamte Vertraglaufzeit zu planen. Auf diese Kosten ist ein Gewinnaufschlag vorzunehmen, der z. B. aus einer Zielumsatzrendite abgeleitet werden kann. Die eigentliche Berechnung der FbH-Rate ergibt sich, wie nachfolgend angegeben, aus der Division der gesamten geplanten Kosten zuzüglich des Gewinnaufschlags durch die gesamte Flugzeit der Triebwerke in der Vertragslaufzeit:

$$
F b H-\text { Rate }=\frac{\sum_{t=1}^{T} \sum_{e=1}^{E} K_{t e}^{p} \cdot\left(1+\frac{r}{(1-r)}\right)}{\sum_{t=1}^{T} \sum_{e=1}^{E} E F_{t e}^{p}}
$$




\begin{tabular}{|c|c|c|c|c|c|c|c|}
\hline \multicolumn{8}{|l|}{$\mathrm{K}_{\mathrm{te}}-$ Mainten } \\
\hline \multicolumn{8}{|c|}{ des e-ten Triebwerks in de } \\
\hline \multicolumn{8}{|c|}{ Zielumsatzrendite } \\
\hline \multicolumn{8}{|l|}{ Indizes } \\
\hline \multicolumn{8}{|c|}{ r Vertrag } \\
\hline \multicolumn{8}{|c|}{ - Index der Triebwerke, $\mathrm{e}=1(1) \mathrm{E}$} \\
\hline \multicolumn{8}{|c|}{ - Plangröße. } \\
\hline & & 2003 & $\begin{array}{ll}2004 \\
\end{array}$ & 2005 & 2006 & 2007 & Gesamt \\
\hline \multicolumn{2}{|l|}{ Anzahl Shop Visits } & 0 & 1 & 2 & 2 & 1 & 6 \\
\hline Kosten je Shop Visit & {$[T \$]$} & 1.080 & 1.080 & 1.080 & 1.080 & 1.080 & \\
\hline Kosten & [T\$] & 0 & 1.080 & 2.160 & 2.160 & 1.080 & 6.480 \\
\hline Gewinnaufschlag & {$[\mathrm{T} \$]$} & & & & & & 720 \\
\hline Gesamter Verrechnungsbetrag & {$[\mathrm{T} \$]$} & & & & & & 7.200 \\
\hline Anzahl Triebwerke & & 6 & 6 & 6 & 6 & 6 & \\
\hline \multicolumn{8}{|l|}{ Flugstunden } \\
\hline je Triebwerk und Jahr & [EFH] & 2.500 & 2.500 & 2.500 & 2.500 & 2.500 & \\
\hline gesamte Flotte pro Jahr & {$[\mathrm{EFH}]$} & 15.000 & 15.000 & 15.000 & 15.000 & 15.000 & 75.000 \\
\hline $\begin{array}{l}\text { Rate je Flugstunde } \\
\end{array}$ & {$[\mathrm{S} / \mathrm{EFH}]$} & & & & & & 96,0 \\
\hline
\end{tabular}

Tab. 3: FbH-Kalkulation mit fiktiven Werten

In Tabelle 3 wird das eingefuihrte Beispiel für einen $\mathrm{FbH}$ Vertrag über fünf Jahre weitergefuihrt. In einem ersten Schritt werden die gesamten Maintenance-Kosten mit einem Gewinnaufschlag versehen, der einer Zielumsatzrendite von $10 \%$ entspricht, so dass sich ein Verrechnungsbetrag von 7,2 Mio. \$ ergibt. Insgesamt beinhaltet der Vertrag sechs Triebwerke, die pro Jahr 2.500 EFH (Engine Flight Hours) und aufsummiert uiber die Vertragslaufzeit 75.000 EFH fliegen. Teilt man den Verrechnungsbetrag durch die gesamten Flugstunden, erhält man eine FbH-Rate von 96 \$ je Flugstunde eines Triebwerks.

Während der Vertragslaufzeit hat der Operator Zahlungen an den MRO-Shop zu leisten, die anhand der Multiplikation der FbH-Rate mit den tatsächlich geflogenen Stunden je Triebwerk berechnet werden. Im Beispiel fliegen die sechs Triebwerke des Operators 15.000 Stunden. Multipliziert mit der FbH-Rate, ergeben sich damit, wie in Tabelle 4 dargestellt, jährliche Zahlungen von 1,44 Mio. \$.

\begin{tabular}{|c|c|c|c|c|c|c|c|}
\hline & & 2003 & 2004 & 2005 & 2006 & 2007 & Total \\
\hline \multicolumn{8}{|l|}{ MRO-Shop } \\
\hline Anzahlungen & [T \$] & 1.440 & 1.440 & 1.440 & 1.440 & 1.440 & 7.200 \\
\hline Erlöse & [T \$] & 0 & 1.080 & 2.160 & 2.160 & 1.800 & 7.200 \\
\hline Kosten & [T\$] & 0 & 1.080 & 2.160 & 2.160 & 1.080 & 6.480 \\
\hline Ergebnis & [T \$] & 0 & 0 & 0 & 0 & 720 & 720 \\
\hline \multicolumn{8}{|l|}{ Operator } \\
\hline Zahlungen & [T \$] & 1.440 & 1.440 & 1.440 & 1.440 & 1.440 & 7.200 \\
\hline
\end{tabular}

Tab. 4: Mehrperiodige Ergebnisse eines FbH-Vertrages mit fiktiven Werten

Nach deutschem Handelsrecht sind diese Zahlungen vom MRO-Shop als erhaltene Anzahlungen zu behandeln, mit denen die einzelnen Shop Visits zu finanzieren sind. Folgt man der Logik, dass Gewinne erst mit Abschluss des gesamten Auftrags zu realisieren sind, werden in der Vertragslaufzeit lediglich Erlöse in Höhe der angefallenen Kosten verbucht und so ein Ergebnis von Null erzielt. Erst mit Ablauf des Vertrags werden der verbleibende Anzahlungsbestand aufgelöst, entsprechende Erlöse gebucht und so das Ergebnis des FbH-Vertrags realisiert. Da im Beispiel die FbH-Rate analog zur T\&M-Kalkulation entsprechend einer Zielumsatzrendite von $10 \%$ geplant wurde, fuihrt auch dieser Vertrag zu einem Ergebnis von 720 T \$.

\subsection{GMC-Verträge}

GMC-Verträge verbinden Aspekte der beiden vorgestellten Vertragsarten. GMC-Verträge sind langfristige und exklusive Verträge über ein definiertes, vom MRO-Shop zu erbringendes Leistungsprogramm. Grundsätzlich wird jeder Shop Visit einzeln auf der Basis Time and Material abgerechnet. Zusätzlich wird dem Operator mit Vertragsabschluss eine Höchstgrenze der fuir ihn anfallenden Maintenance-Kosten zugesichert. Wenn nach Vertragsende die für den Operator angefallenen Kosten die Höchstgrenze überschritten haben, bekommt er einen Teil oder den gesamten Differenzbetrag vom MRO-Shop zurückerstattet. Oft wird bei GMC-Verträgen vereinbart, dass der MRO-Shop $50 \%$ dieses Differenzbetrages übernimmt. ${ }^{13}$

Die Obergrenze der Maintenance-Kosten wird auf der Basis einer Rate je Flugstunde abgebildet, wobei grundsätzlich die gesamten geplanten T\&M-Erlöse durch die gesamten geplanten Flugstunden in der Vertragslaufzeit dividiert werden:

$$
G M C^{p}=\frac{\sum_{t=1}^{T} \sum_{e=1}^{E} E_{t e}^{p}}{\sum_{t=1}^{T} \sum_{e=1}^{E} E F_{t e}^{p}}
$$

mit

GMC - maximale Kosten je Flugstunde und Triebwerk, GMC-Rate

$\mathrm{E}_{\text {te }} \quad-\quad \mathrm{T} \& M$-Erlöse für das e-te Triebwerk in der t-ten Periode

In Tabelle 5 wird das eingeführte Beispiel fuir eine GMCKalkulation weitergeführt. Die Summe der gesamten T\&M-Erlöse wird dem Kunden als Gesamtkosten garantiert. Dividiert man diese Höchstgrenze der Maintenance-Kosten durch die Gesamtsumme der Flugstunden der Triebwerke, erhält man eine Rate von 96 \$ je Flugstunde eines Triebwerks.

\begin{tabular}{lrrrrrrr}
\hline & 2003 & 2004 & 2005 & 2006 & 2007 & Total \\
\hline Anzahl Shop Visits & & 0 & 1 & 2 & 2 & 1 & 6 \\
T\&M-Erlös je SV & {$[T \$]$} & 1.200 & 1.200 & 1.200 & 1.200 & 1.200 & \\
T\&M-Erlös je Periode & {$[T \$]$} & 0 & 1.200 & 2.400 & 2.400 & 1.200 & 7.200 \\
Garantierte Gesamtkosten & {$[T \$]$} & & & & & & $\mathbf{7 . 2 0 0}$ \\
\hline $\begin{array}{l}\text { Anzahl Triebwerke } \\
\text { Flugstunden }\end{array}$ & & 6 & 6 & 6 & 6 & 6 & \\
$\quad$ je Triebwerk und Jahr & {$[\mathrm{EFH}]$} & 2.500 & 2.500 & 2.500 & 2.500 & 2.500 & \\
$\quad$ gesamte Flotte pro Jahr & {$[\mathrm{EFH}]$} & 15.000 & 15.000 & 15.000 & 15.000 & 15.000 & $\mathbf{7 5 . 0 0 0}$ \\
\hline GMC & {$[\$ / E F H]$} & & & & & & $\mathbf{9 6 , 0}$ \\
\hline
\end{tabular}

Tab. 5: GMC-Kalkulation mit fiktiven Werten

Die Überprüfung der Gesamtkosten im Verhältnis zu den garantierten Maintenance-Kosten erfolgt auf der Basis einer zu berechnenden Ist-Rate, indem analog zur GMCRate die T\&M-Erlöse des MRO-Shops durch die geflogenen Stunden der Triebwerke dividiert werden. Die eigentliche Ruickerstattung ergibt sich aus der Differenz der GMC-Rate und der Ist-Rate, die mit den gesamten in der Vertragslaufzeit geflogenen Stunden der Triebwerke und dem Prozentsatz der Kostenbeteiligung des MRO-Shops multipliziert wird: 


$$
\begin{aligned}
& K A= \begin{cases}\left(G M C^{p}-G M C^{r}\right) \cdot \sum_{t=1}^{T} \sum_{e=1}^{E} E F_{t e}^{r} \cdot \alpha & \text { für } \quad\left(G M C^{p}-G M C^{r}\right)<0 \\
0 & \text { sonst }\end{cases} \\
& \text { mit } \\
& \text { KA - Kostenausgleich } \\
& \alpha \quad \text { - Beteiligungsfaktor des MRO-Shops bei Kosten- } \\
& \text { uiberschreitungen }
\end{aligned}
$$

Wenn im Beispiel gemäß Tabelle 5 die Summe der T\&MErlöse des MRO-Shops die Höchstgrenze der garantierten Maintenance-Kosten nicht überschreitet, wird die Ist-Rate kleiner oder gleich der GMC-Rate sein. Deshalb wird im Folgenden davon ausgegangen, dass die realisierten Shop Visits einem höheren Schadensfall unterlagen und so zu 5 \% höheren Kosten gegenüber dem ursprüinglichen Beispiel fuihrten. Entsprechend dem T\&M-Kalkulationsschema in Tabelle 1 wird ein höherer Kostenanfall unmittelbar an den Kunden weitergegeben, so dass auch die T\&M-Erlöse im Beispiel um 5 \% steigen. Dividiert man, wie in Tabelle 6 angegeben, die höheren T\&M-Erlöse von 7,56 Mio. \$ durch die gesamten Flugstunden, ergibt sich eine Ist-Rate von 100,8 \$ je Flugstunde eines Triebwerks. Multipliziert man die daraus resultierende Differenz zur GMC-Rate mit den

\begin{tabular}{|c|c|c|c|c|c|c|c|}
\hline & & 2003 & 2004 & 2005 & 2006 & 2007 & Total \\
\hline \multicolumn{8}{|l|}{ MRO-Shop } \\
\hline T\&M-Erlöse & [T\$] & 0 & 1.260 & 2.520 & 2.520 & 1.260 & 7.560 \\
\hline Kosten & {$[\pi \$]$} & 0 & 1.134 & 2.268 & 2.268 & 1.134 & 6.804 \\
\hline T\&M-Ergebnis & {$[\pi \$]$} & 0 & 126 & 252 & 252 & 126 & 756 \\
\hline Flugstunden der gesamten Flotte & {$[\mathrm{H}]$} & 15.000 & 15.000 & 15.000 & 15.000 & 15.000 & 75.000 \\
\hline Ist-Rate & [\$/EFH] & & & & & & 100,8 \\
\hline Delta Ist-Rate / GMC & [S/EFH] & & & & & & $-4,8$ \\
\hline Kostenausgleich & & & & & & -180 & \\
\hline GMC-Ergebnis & {$[\pi \$]$} & 0 & 126 & 252 & 252 & -54 & 576 \\
\hline \multicolumn{8}{|l|}{ Operator } \\
\hline Kosten & [T\$] & 0 & 1.260 & 2.520 & 2.520 & 1.260 & 7.560 \\
\hline Kostenausgleich & {$[\Pi \$]$} & & & & & -180 & -180 \\
\hline Gesamt & [T\$] & 0 & 1.260 & 2.520 & 2.520 & 1.080 & 7.380 \\
\hline
\end{tabular}
gesamten Flugstunden und einem Beteiligungsfaktor von $50 \%$, erhält man eine Kostenrüickerstattung von 180 T \$. Diese Kostenrïckerstattung mindert aus Sicht des MROShops das Ergebnis des letzten Vertragsjahres und somit des gesamten Vertrages. Für den Operator führt die Erstattung unmittelbar zu einer Ergebnisverbesserung.

Tab. 6: Mehrperiodige Ergebnisse eines GMC-Vertrages mit fiktiven Werten

Auch für GMC-Verträge existiert eine Anzahl von Variationen. So können einzelne Rechnungspositionen mit einem Festpreis verbunden sein. Eine andere Variante besteht darin, dass nicht für die gesamte Laufzeit des Vertrages eine GMC-Rate angegeben wird, sondern spezifisch fuir die Zeiträume zwischen den einzelnen geplanten Shop Visits. Mit dieser so genannten GMC-R (Guaranteed Maintenance Costs per Run) wird eine Obergrenze der Maintenance-Kosten eines Triebwerks für die Zeit zwischen den einzelnen geplanten Shop Visits vom MRO-Shop garantiert.

\section{Risikostrukturen der Vertragsformen}

Im Rahmen der bisherigen Ausfuihrungen wurde im Wesentlichen davon ausgegangen, dass die für die einzelnen Vertragsarten geplanten Ergebnisse mit Sicherheit eintreten werden. Diese Prämisse kann für die Realität in der Maintenance von zivilen Flugtriebwerken nicht aufrechterhalten werden, da die Ergebnisse derartiger Verträge durch eine Vielzahl interner und externer, teilweise nicht prognostizierbarer Störgrößen beeinflusst werden. Aus dem Auftreten derartiger Störgrößen können letztlich Ergebnisse für den Operator bzw. den MROShop resultieren, die von den vor Vertragsabschluss geplanten Ergebnissen abweichen.

\subsection{Ausgewählte Ursachen fuir Chancen und Risiken}

Aus der Menge der möglichen Ursachen für derartige Abweichungen ${ }^{14}$ lassen sich z. B. der Anteil der zu verschrottenden Teile (Scrap Rates) sowie die Anzahl der ungeplanten Shop Visits nennen. ${ }^{15}$ Beide stellen Parameter fuir die Kalkulation bzw. Planung eines Vertrages dar und sind in diesem Zusammenhang nicht mit Sicherheit zu prognostizieren. So führt eine Veränderung der Scrap Rates unmittelbar zu einer veränderten Ausprägung der Kosten für auszutauschende Teile. Zur Prognose ungeplanter Shop Visits wird oft der Anteil der Triebwerke einer Flotte mit ungeplanten Ereignissen geschätzt. Wenn sich dieser Anteil verändert, tritt eine gegenuiber der Vertragsplanung abweichende Anzahl ungeplanter Shop Visits und letztlich eine andere Ausprägung der Maintenance-Kosten der betrachteten Flotte auf. Für die beiden genannten Risikoparameter könnten grundsätzlich im Rahmen der Planung der Ergebnisse der einzelnen Vertragsformen entsprechende Verteilungen mit Erwartungswert und Varianz auf der Basis empirischer Triebwerksdaten geschätzt werden, wobei im Allgemeinen unterstellt werden kann, dass sich diese Parameter normalverteilt verhalten. In Abhängigkeit dieser Zufallsvariablen würden auch die geplanten Ergebnisse der Vertragsarten einer Wahrscheinlichkeitsverteilung unterliegen.

Für die weiteren Betrachtungen wird das eingefuihrte Beispiel gemäß Tabelle 7 weitergeführt. Es wird unterstellt, dass die Maintenance-Kosten der einzelnen im Beispiel betrachteten geplanten Shop Visits unsicher sind, wobei aus Vereinfachungsgründen lediglich drei verschiedene Schadensfälle betrachtet werden. Der mittlere Schadensfall entspricht dem bisherigen Beispiel und repräsentiert den Erwartungswert der Kosten der Shop Visits und der daraus resultierenden Ergebniseffekte. Der geringe Schadensfall soll den Fall repräsentieren, dass ein um $5 \%$ geringerer Kostenanfall zu verzeichnen ist. Der hohe Schadensfall fuihrt zu einem fünfprozentig höheren Kostenanfall gegenuiber dem Normalfall.

\begin{tabular}{lrrrrrrr}
\hline & 2003 & 2004 & 2005 & 2006 & 2007 & Gesamt \\
\hline $\begin{array}{l}\text { Anzahl Shop Visits } \\
\text { Kosten }\end{array}$ & 0 & 1 & 2 & 2 & 1 & 6 \\
$\quad$ geringer Schadensfall & {$[\mathrm{T} \$]$} & 0 & 1.026 & 2.052 & 2.052 & 1.026 & $\mathbf{6 . 1 5 6}$ \\
$\quad$ mittlerer Schadensfall & {$[\mathrm{T} \$]$} & 0 & 1.080 & 2.160 & 2.160 & 1.080 & $\mathbf{6 . 4 8 0}$ \\
$\quad$ hoher Schadensfall & {$[\mathrm{T} \$]$} & 0 & 1.134 & 2.268 & 2.268 & 1.134 & $\mathbf{6 . 8 0 4}$ \\
\hline
\end{tabular}

Tab. 7: Fiktive Schadensfälle

Interpretiert man den Anfall der Schadensfälle als einen Zustandsraum mit drei Umweltzuständen und die drei Vertragsformen als zu wählende Aktionen, kann jeder Kombination von Aktion und Umweltzustand ein Ergebnis entsprechend den dargestellten Kalkulationen zugeordnet werden. 


\subsection{Chancen und Risiken für den MRO-Shop}

Die Chancen und Risiken für den Shop ergeben sich unmittelbar aus den Ergebnisrealisierungen der Vertragsformen im Rahmen der Menge der möglichen Schadensfälle.

Da die Konditionen eines T\&M-Vertrages von den Schadensfällen unabhängig sind und alle Kostenbestandteile an den Operator weiterberechnet werden, beinhaltet ein solcher Vertrag für den MRO-Shop keine Risiken im Sinne von überproportionalen Ergebnisschmälerungen bei hohen Schadensfällen, aber auch keine Chancen auf überproportional steigende Ergebnisse bei geringen Schadensfällen. So führen die jeweils um $5 \%$ variierenden Schadensfälle des in Tabelle 8 weitergefuihrten Beispiels gegenüber dem mittleren Schadensfall zu um $5 \%$ fallenden bzw. steigenden absoluten Ergebnissen, wobei die Umsatzrendite konstant $10 \%$ beträgt.

\begin{tabular}{l|ccc}
\hline MRO-Shop & $\begin{array}{c}\text { geringer } \\
\text { Schadensfall }\end{array}$ & $\begin{array}{c}\text { mittlerer } \\
\text { Schadensfall }\end{array}$ & $\begin{array}{c}\text { hoher } \\
\text { Schadensfall }\end{array}$ \\
\hline Time and Material & 684 & 720 & 756 \\
GMC & 684 & 720 & 576 \\
FbH & 1.044 & 720 & 396 \\
\hline
\end{tabular}

Tab. 8: Unsichere Ergebnisse für den MRO-Shop nach Vertragsform und Schadensfall

Solange die dem Operator in Rechnung gestellten T\&MErlöse die garantierte Obergrenze nicht überschreiten, verhält sich die Ergebnissituation eines GMC-Vertrages für den MRO-Shop analog zu der eines T\&M-Vertrages. Im Beispiel entspricht das dem geringen und dem mittleren Schadensfall. Sobald (gemessen an der GMC-Rate) diese Grenze nicht mehr eingehalten wird, steht dem Operator ein Kostenausgleich zu, der aus Sicht des MROShops zu einer Ergebnisreduzierung fuihrt. Wie in Tabelle 6 dargestellt, wird im Beispiel bei dem hohen Schadensfall die GMC-Rate uiberschritten und der MRO-Shop hat einen Anteil von $50 \%$ des Differenzbetrages zu tragen. Damit beinhaltet der GMC-Vertrag bei diesem Schadensfall ein um den Kostenausgleich reduziertes Ergebnis gegenüber dem T\&M-Vertrag. Der MRO-Shop übernimmt daher bei einem derartigen Vertrag entsprechend seinem Beteiligungsfaktor bei Überschreitungen der garantierten Maintenance-Kosten einen Teil des Risikos bei einem Anfall hoher Schadensfälle.

FbH-Verträge besitzen aufgrund ihrer konstanten Rate je Triebwerksflugstunde einen Festpreischarakter. Demzufolge hat jede Veränderung der Maintenance-Kosten der Shop Visits einen unmittelbaren Einfluss auf das Ergebnis eines solchen Vertrages. Für hohe Schadensfälle, deren Kosten nicht von der FbH-Rate gedeckt sind, übernimmt der MRO-Shop das volle Risiko. Allerdings kann der MRO-Shop alle Ergebniseffekte realisieren, die auf einem niedrigeren Kostenanfall aufgrund geringerer Schadensfälle bei konstanten Zahlungen des Operators basieren.

Die Risikostrukturen, im Sinne der Übernahme von Risiken und der Möglichkeit der Realisierung von Ergebnischancen, sind aus Sicht des MRO-Shops in Tabelle 9 nochmals zusammengefasst.

\begin{tabular}{l|cc}
\hline MRO-Shop & Chancen & Risiken \\
\hline Time and Material & $0 \%$ & $0 \%$ \\
GMC mit $\alpha=50 \%$ & $0 \%$ & $50 \%$ \\
FbH & $100 \%$ & $100 \%$ \\
\hline
\end{tabular}

Tab. 9: Struktur der Chancen und Risiken für den MRO-Shop nach Vertragsformen

\subsection{Chancen und Risiken für den Operator}

Die Chancen und Risiken für den Operator ergeben sich einerseits aus den auftretenden Schadensfällen und andererseits aus der Art der Rechnungsstellung des MROShops. Grundsätzlich verfolgt der Operator ein Kostenminimierungsziel hinsichtlich der vom MRO-Shop einstehenden Maintenance-Kosten. Auftretende Risiken führen tendenziell zu steigenden und Chancen zu sinkenden Maintenance-Kosten.

Da der MRO-Shop dem Operator alle Kosten eines Shop Visits unmittelbar in Rechnung stellt, variieren die Maintenance-Kosten des Operators mit den einzelnen Schadensfällen. Damit trägt der Operator alle Risiken eines solchen Vertrags, da mit höheren Schadensfällen seine Maintenance-Kosten steigen. Allerdings kann er auch die Chancen eines solchen Vertrages realisieren, da sich mit geringeren Schadensfällen gleichzeitig seine Ergebnissituation im Sinne geringerer Maintenance-Kosten verbessert. In Tabelle 10 sind für das Beispiel die unsicheren Kosten der einzelnen Vertragstypen gegeben. Im Falle eines T\&M-Vertrages führen die jeweils um $5 \%$ variierten Kosten entsprechend der in Tabelle 1 gegebenen Konditionen eines solchen Vertrages zu um jeweils $5 \%$ veränderten Time and Material-Rechnungen des MROShops an den Operator.

\begin{tabular}{l|ccc}
\hline Operator & $\begin{array}{c}\text { geringer } \\
\text { Schadensfall }\end{array}$ & $\begin{array}{c}\text { mittlerer } \\
\text { Schadensfall }\end{array}$ & $\begin{array}{c}\text { hoher } \\
\text { Schadensfall }\end{array}$ \\
\hline Time and Material & 6.840 & 7.200 & 7.560 \\
GMC & 6.840 & 7.200 & 7.380 \\
FbH & 7.200 & 7.200 & 7.200 \\
\hline
\end{tabular}

Tab. 10: Unsichere Kosten für den Operator nach Vertragsform und Schadensfall

Für geringe und mittlere Schadensfälle verhält sich die Ergebnissituation eines GMC-Vertrages analog zu der eines T\&M-Vertrages. Wenn beim Auftritt hoher Schadensfälle die vom MRO-Shop in Rechnung gestellten Maintenance-Kosten die garantierte Obergrenze überschreiten, steht dem Operator ein Kostenausgleich zu. Damit trägt der Operator bei einem GMC-Vertrag entsprechend des Beteiligungsfaktors des MRO-Shops bei Kostenuiberschreitungen nur einen Teil des Risikos. Im Beispiel sind daher die Maintenance-Kosten des GMC-Vertrages für den geringen und den mittleren Schadensfall identisch zu denen des T\&M-Vertrages, während für den hohen Schadensfall der in Tabelle 6 berechnete Kostenausgleich von $180 \mathrm{~T}$ Euro zu einer entsprechenden Verringerung der Maintenance-Kosten gegenüber den Kosten des T\&M-Vertrages führt. 
Bei einem FbH-Vertrag zahlt der Operator eine konstante, zum Vertragsbeginn vereinbarte Rate je Flugstunde. Die tatsächlich eintretenden Schadensfälle haben somit keinen Einfluss auf die Ergebnissituation des Operators. Damit trägt der Operator bei Abschluss eines derartigen Vertrages kein Risiko, besitzt aber weiterhin keine Möglichkeit, die Chancen geringer Schadensfälle ergebnisverbessernd zu realisieren.

Die genannten Risikostrukturen sind nochmals in Tabelle 11 zusammengefasst. Diese Strukturen stellen das Gegenstück zu den Chancen und Risiken der Vertragsformen für einen MRO-Shop dar. Bei einem T\&M-Vertrag übernimmt der Operator im Gegensatz zum MRO-Shop alle Chancen und Risken. Ein GMC-Vertrag hat zur Folge, dass der Operator alle Chancen realisieren kann und sich das Risiko hoher Schadensfälle mit dem MRO-Shop teilt. Bei Abschluss eines FbH-Vertrages trägt der MROShop alle Chancen und Risiken, so dass die MaintenanceKosten für den Operator sichere Größen darstellen.

\begin{tabular}{l|cc}
\hline Operator & Chancen & Risiken \\
\hline Time and Material & $100 \%$ & $100 \%$ \\
GMC mit $\alpha=50 \%$ & $100 \%$ & $50 \%$ \\
FbH & $0 \%$ & $0 \%$ \\
\hline
\end{tabular}

Tab. 11: Struktur der Chancen und Risiken für den Operator nach Vertragsformen

\section{Wahl einer Vertragsform gemäß den Risikostrukturen}

Die aus den Risikostrukturen resultierenden unsicheren Ergebnisse führen dazu, dass bei der Wahl einer Vertragsform die Sicherheitspräferenzen entsprechender Entscheidungsträger einzubeziehen sind. Wenn für den Eintritt der unsicheren Ergebnisse Wahrscheinlichkeitsverteilungen ermittelbar sind, liegt hinsichtlich der zu wählenden Vertragsform eine Entscheidung unter Risiko unter Beachtung der Risikopräferenzen der Entscheidungsträger vor. ${ }^{16}$ Da die konkrete Ermittlung der Wahrscheinlichkeitsverteilungen aus praktischer Sicht nicht immer möglich ist, erscheint die Anwendung derartiger Entscheidungsregeln problematisch.

Grundsätzlich können aber auch ohne Angabe von Wahrscheinlichkeiten in Abhängigkeit der Sicherheitspräferenzen der Entscheidungsträger allgemeine Aussagen uiber die relative Vorteilhaftigkeit der einzelnen Vertragsarten getroffen werden, die auch für eine konkrete Entscheidungssituation anwendbar sind. Für die weiteren Betrachtungen werden daher die Entscheidungsträger entsprechend ihren Einstellungen hinsichtlich unsicherer Ergebnisse in Pessimisten, Neutrale und Optimisten unterschieden. Ein Pessimist bewertet die Risiken einer Vertragsform höher als die Chancen. Ein neutraler Entscheider schätzt die Chancen und Risiken einer Vertragsform gleich stark ein. Ein Optimist beurteilt die Chancen einer Vertragsform höher als die entsprechenden Risken.

\subsection{Rangfolge der Vertragsformen für den MRO-Shop}

Ein Pessimist bewertet jede Übernahme eines Risikos als potenzielle Ergebnisschmälerung, die seinem grundsätzlichen Ziel der Ergebnismaximierung eines Vertrages entgegensteht. In diesem Sinn wird sich ein solcher Entscheidungsträger in erster Linie für einen T\&M-Vertrag entscheiden, da dieser keine Risiken für den MROShop beinhaltet. Weiterhin wird er einen GMC-Vertrag einem FbH-Vertrag immer dann vorziehen, wenn der Beteiligungsfaktor des MRO-Shops bei Kostenüberschreitungen einen Wert kleiner als 100 Prozent besitzt, weil der MRO-Shop in diesem Fall im Gegensatz zu einem FbH-Vertrag nur einen Teil des Risikos übernimmt.

Ein neutraler Entscheider bewertet die Chancen und Risiken einer Vertragsform gleich stark. Insofern die Ergebniswirkungen der Schadensfälle normalverteilt sind, ist es für diesen Entscheidungsträger irrelevant, ob er bei einem FbH-Vertrag 100 Prozent der Chancen und Risiken trägt oder dass bei einem T\&M-Vertrag keine Chancen bzw. Risiken vorliegen. Daher besteht fuir beide Vertragformen eine Entscheidungsindifferenz. Diese beiden gleich vorteilhaften Vertragsformen werden von einem neutralen Entscheider immer vorteilhafter als ein GMC-Vertrag bewertet. Diese Einschätzung liegt vor allem darin begründet, dass bei diesem Vertrag keine Chancen existieren, aber immer eine Risikoübernahme vorliegt und damit immer ein geringeres Ergebnis als für die beiden anderen Vertragsformen zu erwarten ist.

Ein optimistischer Entscheidungsträger wird immer einen FbH-Vertrag allen anderen Vertragsformen vorziehen, da nur ein FbH-Vertrag die Chancen für eine Ergebnisverbesserung beinhaltet. Weiterhin ist für einen Optimisten ein T\&M-Vertrag gegenuiber einem GMC-Vertrag vorteilhafter, da dieser von keiner Risikoübernahme betroffen ist und damit bei niedrigen und mittleren Schadensfällen zu identischen sowie bei hohen Schadensfällen zu besseren Ergebnissen als ein GMC-Vertrag fuihrt.

Diese in Tabelle 12 zusammengefassten Rangfolgen lassen sich für das Beispiel anhand eines einfachen Entscheidungskriteriums nachweisen.

\begin{tabular}{l|c|c|c}
\hline MRO-Shop & Pessimist & Neutral & Optimist \\
\hline Time and Material & 1 & 1 & 2 \\
GMC mit $\alpha=50 \%$ & 2 & 2 & 3 \\
FbH & 3 & 1 & 1 \\
\hline
\end{tabular}

Tab. 12: Rangfolge der Vertragsformen für einen MRO-Shop

Für das Beispiel mit seinen drei Schadensfällen, für die keine Eintrittswahrscheinlichkeiten angegeben sind, lässt sich die so genannte Pessimismus-Optimismus-Regel (Hurwicz-Kriterium) anwenden. ${ }^{17}$ Dazu sollen in einem ersten Schritt die einzelnen auszuwählenden Vertragsformen als Elemente eines Aktionsraumes interpretiert werden:

$A=\left\{a_{i} \mid i=1(1) 3\right\}$ 
mit

A - Aktionsraum

$\mathrm{a}_{\mathrm{i}} \quad-$ Aktion i (Vertragsform i)

Index

$\mathrm{i} \quad$ - Index der Aktionen, $\mathrm{i}=1(1) 3$.

In Abhängigkeit der einzelnen Schadensfälle, die im Weiteren mit $\mathrm{j}=1(1) 3$ indiziert werden, kann für jede Aktion i ein spezifisches Ergebnis $\mathrm{e}_{\mathrm{ij}}$ geplant werden. Verfolgt ein Entscheidungsträger ein Maximierungsziel, kann das Hurwicz-Kriterium wie folgt formuliert werden:

$$
\begin{aligned}
& \Phi\left(a_{i}\right)=\lambda \cdot \max _{j}\left(e_{i j}\right)+(1-\lambda) \cdot \min _{j}\left(e_{i j}\right) ; i=1(1) 3 ; 0 \leq \lambda \leq 1 \\
& \Phi^{*}=\max _{i} \Phi\left(a_{i}\right) \\
& \text { mit } \\
& \Phi\left(\mathrm{a}_{\mathrm{i}}\right) \text { - Pessimismus-Optimismus-Nutzenwert der i-ten }
\end{aligned}
$$

Es erfolgt demnach mit dem Hurwicz-Kriterium eine Wichtung der Extremwerte einer Vertragsform anhand des Pessimismus-Optimismus-Indexes. Die Präferenzen eines Optimisten wird mit einem Pessimismus-Optimismus-Index $\lambda$ größer 0,5 abgebildet. Ein solcher Entscheider bewertet damit das maximale Ergebnis einer Vertragsform höher als das minimale, da dieses ungünstige Ergebnis mit (1- $\lambda)$ multipliziert wird. Ein neutraler Entscheider beurteilt die Chancen und Risiken einer Vertragsform gleich, was einem Pessimismus-Optimismus-Index $\lambda$ von 0,5 entspricht. Ein Pessimist wichtet die Risiken und damit das ungünstige minimale Ergebnis einer Vertragsform höher als die Chancen dieses Vertrags. Daher wird dieser Entscheider einen PessimismusOptimismus-Index $\lambda$ mit einer Ausprägung kleiner 0,5 angeben. Letztlich wird ein Entscheidungsträger die Vertragsform wählen, die gemäß dem Hurwicz-Kriterium zu dem höchsten Nutzenwert führt.

Wendet man das Hurwicz-Kriterium für das gegebene Beispiel aus Sicht des MRO-Shops und die drei Gruppen von Entscheidungsträgern an, erhält man die in Tabelle 13 angegebenen Ergebnisse. In dieser Tabelle sind dazu für die einzelnen Arten von Entscheidungsträgern die

\begin{tabular}{|c|c|c|c|c|c|c|c|c|c|}
\hline \multirow[t]{2}{*}{ MRO-Shop } & \multirow[t]{2}{*}{$\begin{array}{c}\text { geringer } \\
\text { Schadensfall }\end{array}$} & \multirow[t]{2}{*}{$\begin{array}{c}\text { mittlerer } \\
\text { Schadensfall }\end{array}$} & \multirow[t]{2}{*}{$\begin{array}{c}\text { hoher } \\
\text { Schadensfall }\end{array}$} & \multicolumn{2}{|c|}{$\begin{array}{c}\text { Pessimist } \\
\lambda=0,25\end{array}$} & \multicolumn{2}{|c|}{$\begin{array}{l}\text { Neutral } \\
\lambda=0,5\end{array}$} & \multicolumn{2}{|c|}{$\begin{array}{l}\text { Optimist } \\
\lambda=0,75\end{array}$} \\
\hline & & & & $\Phi$ & Rang & $\Phi$ & Rang & $\Phi$ & Rang \\
\hline Time and Material & 684 & 720 & 756 & 702 & (1) & 720 & (1) & 738 & (2) \\
\hline GMC & 684 & 720 & 576 & 612 & (2) & 648 & (2) & 684 & (3) \\
\hline $\mathrm{FbH}$ & 1.044 & 720 & 396 & 558 & (3) & 720 & (1) & 882 & (1) \\
\hline
\end{tabular}
jeweiligen Pessimismus-Optimismus-Indizes angeben und in Abhängigkeit der einzelnen Vertragsformen die Pessimismus-Optimismus-Nutzenwerte sowie in Klammern die entsprechenden Rangfolgen in Abhängigkeit der Ausprägung der absoluten Nutzenwerte.

Tab. 13: Rangfolge der Vertragsformen für den MRO-Shops gemäß dem Hurvicz-Kriterium

Wie man an den angegebenen Rangfolgen im Beispiel sieht, fuihrt das Hurwicz-Kriterium zu identischen Rangfolgen hinsichtlich der einzelnen Vertragsformen wie die zuvor allgemein erarbeiteten Rangfolgen.

\subsection{Rangfolge der Vertragsformen für den Operator}

Analog zu den MRO-Shops lassen sich allgemeine Aussagen treffen, welche Vertragsformen für einen pessimistischen, einen neutralen und einen optimistischen Entscheidungsträger aus Sicht des Operators vorteilhaft sind.

Ein pessimistischer Entscheider sieht jede Übernahme eines Risikos als potenzielle Ergebnisverschlechterung an, die er stärker als die Chancen potenzieller Ergebnisverbesserungen im Sinne von Kostensenkungen aufgrund geringerer Schadensfälle bewertet. Daher wird ein solcher Entscheider einen FbH-Vertrag allen anderen Vertragsformen vorziehen, da der Operator in diesem Fall kein Risiko übernehmen muss. In der Rangfolge eines derartigen Operators folgt der GMC-Vertrag, da er bei dieser Vertragsform im Gegensatz zu einem T\&M-Vertrag lediglich einen Teil des Risikos trägt.

Ein neutraler, die Chancen und Risiken einer Vertragsform gleich bewertender Entscheidungsträger ist hinsichtlich eines T\&M-Vertrages und eines FbH-Vertrages indifferent in seiner Entscheidung, da es fuir ihn irrelevant ist, ob er von den in ihrer Ergebniswirkung gleich ausgeprägten Chancen und Risiken $0 \%$ oder $100 \%$ übernimmt. Für einen solchen Entscheider ist es rational, sich in erster Linie für einen GMC-Vertrag zu entscheiden, da fuir diese Vertragform aus Sicht des Operators die Chancen stärker als die Risiken im Sinne ihres Potenzials auf Ergebnisverbesserungen bzw. -verschlechterungen ausgeprägt sind.

Ein optimistischer Operator wird die Vertragsformen anstreben, bei denen er die Chance auf eine Ergebnisverbesserung besitzt. In diesem Sinn stehen FbH-Verträge in der Rangfolge der Vertragsformen an letzter Stelle, da sie keine Ergebnisverbesserungspotenziale in Abhängigkeit von den Schadensfällen beinhalten. Von den verbleibenden Vertragsformen wird sich ein optimistischer Entscheider in erster Linie für einen GMC-Vertrag entscheiden, da diese Vertragsform eine 100-prozentige Chance auf Ergebnisverbesserungen verspricht, aber nur ein Teil des Risikos übernommen werden muss.

Die Rangfolgen der Vertragsformen für die einzelnen Typen von Entscheidungsträgern eines Operators sind in Tabelle 14 zusammengefasst und sollen im Weiteren für das Beispiel anhand des Hurwicz-Kriteriums nachgewiesen werden.

\begin{tabular}{l|c|c|c}
\hline Operator & Pessimist & Neutral & Optimist \\
\hline Time and Material & 3 & 2 & 2 \\
GMC mit $\alpha=50 \%$ & 2 & 1 & 1 \\
FbH & 1 & 2 & 3 \\
\hline
\end{tabular}

Tab. 14: Rangfolge der Vertragsformen für einen Operator

Unter Beachtung eines grundsätzlichen Minimierungsziels lässt sich das Hurwicz-Kriterium wie folgt formulieren:

$\Gamma\left(a_{i}\right)=\lambda \cdot \max _{j}\left(e_{i j}\right)+(1-\lambda) \cdot \min _{j}\left(e_{i j}\right) ; i=1(1) 3 ; 0 \leq \lambda \leq 1$

$\Gamma^{*}=\min _{i} \Gamma\left(a_{i}\right)$ 
mit $\begin{aligned} \Gamma\left(\mathrm{a}_{\mathrm{i}}\right)- & \text { Pessimismus-Optimismus-Nutzenwert der i-ten } \\ & \text { Vertragsform des Operators }\end{aligned}$

Es existieren wiederum drei Aktionen $\mathrm{a}_{\mathrm{i}}$ in Form der zur Wahl stehenden Vertragsformen, die in Abhängigkeit der drei Schadensfälle $\mathrm{j}=1(1) 3 \mathrm{zu}$ spezifischen Ausprägungen der Maintenance-Kosten $\mathrm{e}_{\mathrm{ij}}$ fuihren. Es werden erneut die Extremwerte einer Vertragsform mit dem Pessimismus-Optimismus-Index gewichtet, aber in einem zweiten Schritt nicht die Aktion mit dem maximalen, sondern die mit dem minimalen Pessimismus-Optimismus-Nutzenwert als die optimale Vertragsform ausgewählt. Weiterhin sind die Ausprägungen des Pessimismus-Optimismus-Indexes anders zu interpretieren. Ein Pessimist wird nun die maximale Ausprägung der MaintenanceKosten stärker als das entsprechende Minimum bewerten. Damit wird ein Pessimist einen Pessimismus-Optimismus-Index $\lambda$ mit einen Wert größer 0,5 wählen. Neutrale Entscheider, die die Chancen und Risiken gleich gewichtet ansehen, wählen einen Pessimismus-Optimismus-Index $\lambda$ mit einem Wert gleich 0,5 . Optimistische Entscheider, die auf die Chancen orientiert sind, werden das Minimum der Maintenance-Kosten einer Vertragsform stärker gewichten als das entsprechende Maximum. Für diese Typen von Entscheidern gilt daher ein Pessimismus-Optimismus-Index $\lambda$, der eine Ausprägung kleiner als 0,5 besitzt.

Angewendet auf das Beispiel fuihrt das Hurwicz-Kriterium aus Sicht des Operators zu den in Tabelle 15 enthaltenen Ergebnissen.

\begin{tabular}{|c|c|c|c|c|c|c|c|c|c|}
\hline \multirow[t]{2}{*}{ Operator } & \multirow[t]{2}{*}{\begin{tabular}{|c|} 
geringer \\
Schadensfall
\end{tabular}} & \multirow[t]{2}{*}{$\begin{array}{c}\text { mittlerer } \\
\text { Schadensfall }\end{array}$} & \multirow[t]{2}{*}{$\begin{array}{c}\text { hoher } \\
\text { Schadensfall }\end{array}$} & \multicolumn{2}{|c|}{$\begin{array}{l}\text { Pessimist } \\
\lambda=0,75\end{array}$} & \multicolumn{2}{|c|}{$\begin{array}{l}\text { Neutral } \\
\lambda=0,5\end{array}$} & \multicolumn{2}{|c|}{$\begin{array}{l}\text { Optimist } \\
\lambda=0,25\end{array}$} \\
\hline & & & & $\Gamma$ & Rang & $\Gamma$ & Rang & $\Gamma$ & Rang \\
\hline Tim & 6.840 & 7.200 & 7.560 & 7.380 & (3) & 7.200 & (2) & 7.020 & (2) \\
\hline GMC & 6.840 & 7.200 & 7.380 & 7.245 & (2) & 7.110 & (1) & 6.975 & (1) \\
\hline $\mathrm{FbH}$ & 7.200 & 7.200 & 7.200 & 7.200 & (1) & 7.200 & (2) & 7.200 & (3) \\
\hline
\end{tabular}

Tab. 15: Rangfolge der Vertragsformen für den Operator gemäß dem Hurwicz-Kriterium

Die jeweiligen Typen von Entscheidungsträgern wählen jeweils die Vertragsform aus, die den minimalen Pessimismus-Optimismus-Nutzenwert besitzt. Versieht man die einzelnen Vertragsformen entsprechend ihrer Pessimismus-Optimismus-Nutzenwerte mit einer Rangfolge, erkennt man, dass die Anwendung des Hurwicz-Kriteriums für dieses Beispiel zu identischen, wie die zuvor allgemein erarbeiteten Rangfolgen führt.

\subsection{Gemeinsame Vertragswahl}

Bisher wurden die Rangfolgen der Vorteilhaftigkeit von Vertragsformen lediglich separat für den Operator bzw. den MRO-Shop erörtert. Diese Rangfolgen sind immer dann relevant, wenn einer der beiden Vertragspartner bei ausreichender Marktmacht die Vertragsform explizit vorgibt.

Wenn Verhandlungsspielräume hinsichtlich der Vertragsformen bestehen, ist von den beiden potenziellen Vertragspartnern eine Vertragsform gemeinsam zu wählen, wobei hinsichtlich der Rangfolgen gleich- oder gegenläufige Kombinationen bestehen können. So besitzen ein pessimistischer Operator und ein optimistischer MROShop eine eindeutige Präferenz für einen FbH-Vertrag, so dass die Wahl der Vertragsform eindeutig ist. Es können aber auch grundsätzlich konträre Rangfolgen der Vertragsformen existieren. Beispielsweise wird der von dem pessimistischen Operator bevorzugte FbH-Vertrag von einem pessimistischen MRO-Shop als unattraktivste Vertragsform angesehen. Es besteht somit die Notwendigkeit, einen Kompromiss zwischen den beiden potenziellen Vertragspartnern herbeizufuihren. Eine einfache Herangehensweise anhand der Rangfolgen der Vertragsformen des Operators und des MRO-Shops ist problematisch, da diese nicht die negativen monetären Ergebniseffekte bei einem Abgehen von der am stärksten präferierten Vertragsform abbilden. Es wird daher im Folgenden ein Modell zur Kompromissbildung erarbeitet, das diese Effekte beinhaltet.

Die Aktionen dieses Modells sind durch die drei Vertragformen $\mathrm{a}_{\mathrm{i}}$ gegeben, für die die Pessimismus-OptimismusNutzenwerte des MRO-Shops und des Operators gegeben sind. Wenn einer der beiden potenziellen Vertragspartner eine andere als die von ihm am stärksten bevorzugte Vertragsform realisieren muss, entsteht ein monetärer Disnutzen. Dieser kann aus der Differenz des Pessimismus-Optimismus-Nutzenwertes der optimalen Vertragsform und dem Pessimismus-Optimismus-Nutzenwertes der anderen gewählten Vertragsform ermittelt werden. Der Kompromiss besteht darin, dass die Vertragsform ausgewählt wird, bei der die Summe der Disnutzen des Operators und des MRO-Shops einen minimalen Wert annimmt, wobei die Disnutzen der beiden potenziellen Vertragspartner mit einem Einflussparameter $\beta$ gewichtet werden. Dieser Einflussparameter, der nur Werte zwischen 0 und 1 annehmen kann, soll die Verhandlungsstärke des Operators abbilden. Die Stärke des Einflusses des MRO-Shops ergibt sich dann aus dem Wert (1- $\beta$ ). Damit kann ein Modell zur Kompromissbildung wie folgt formuliert werden:

$$
\begin{aligned}
\mathrm{X}\left(a_{i}\right) & =\beta \cdot\left(\Gamma\left(a_{i}\right)-\min _{i} \Gamma\left(a_{i}\right)\right)+(1-\beta) \cdot\left(\max _{i} \Phi\left(a_{i}\right)-\Phi\left(a_{i}\right)\right) ; i=1(1) 3 ; 0 \leq \beta \leq 1 \\
\mathrm{X}^{*} & =\min _{i} \mathrm{X}\left(a_{i}\right)
\end{aligned}
$$

mit

$\mathrm{X}\left(\mathrm{a}_{\mathrm{i}}\right)$ - Kompromiss-Disnutzen der i-ten Vertragsform $\beta-$ Einflussparameter des Operators.

Wendet man dieses Kompromissmodell auf das gegebene Beispiel an, erhält man die in Tabelle 16 dargestellten

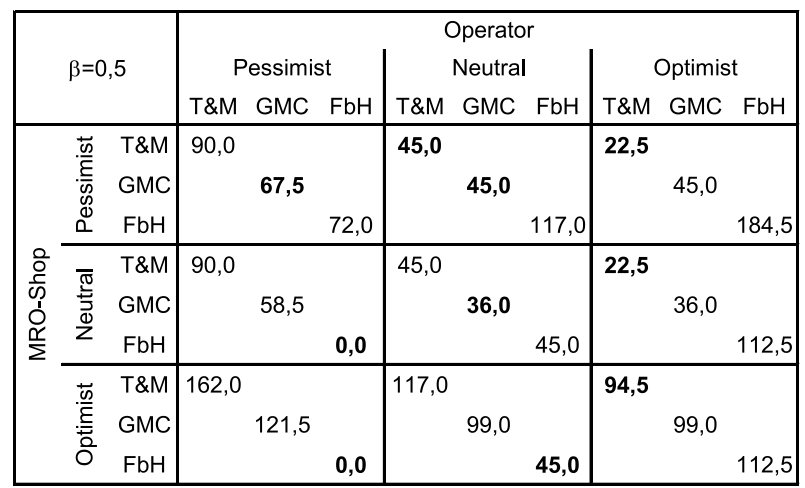

Tab. 16: Disnutzen von Vertragskombinationen bei Gewichtung des Einflusses der beiden Vertragspartner 
Disnutzenwerte, wobei mit einem $\beta$ von 0,5 ein gleich starker Einfluss beider potenziellen Vertragspartner unterstellt wurde. Für die einzelnen Kombinationen der Typen von Entscheidungsträgern kann nun jeweils die Vertragsform ausgewählt werden, die den minimalen gewichteten Disnutzen besitzt.

Da z. B. bei der Kombination pessimistischer Operator/ optimistischer MRO-Shop der FbH-Vertrag bei beiden potenziellen Vertragspartnern die Rangfolge 1 einnimmt, muss der gewichtete Disnutzen des FbH-Vertrags den Wert Null annehmen. Wie schon diskutiert, besteht das Problem der gegenläufigen Rangfolgen der einzelnen Vertragsformen, wenn z. B. beide potenziellen Vertragspartner pessimistisch eingestellt sind. Gemäß dem minimalen gewichteten Disnutzen besteht fuir diese Kombination in einem GMC-Vertrag der optimale Kompromiss.

In diesem Sinn kann für alle Kombinationen nach Typen von Entscheidungsträgern die jeweils optimale Vertragsform ermittelt werden. In Tabelle 17 sind für diese Kombinationen die entsprechenden optimalen Vertragsformen abgebildet, wobei drei unterschiedliche Fälle der Stärke des Einflusses beider Vertragspartner betrachtet wurden. Bei einem $\beta$ von 0,5 besitzen beide einen identischen Einfluss, so dass dieser Fall eine grundsätzliche Kompromisssituation abbildet. Je stärker der Operator bei einem $\beta$ größer 0,5 oder der MRO-Shop bei einem $\beta$ kleiner 0,5 einen Einfluss auf die Wahl der Vertragsform besitzt, werden sich die entsprechend auszuwählenden Vertragsformen den isoliert betrachteten Rangfolgen des Operators bzw. des MRO-Shops annähern. In diesem Sinn kann die separate Rangfolge des Operators als ein Extremfall dieses Modells mit einem $\beta$ gleich 1 und die separate Rangfolge des MRO-Shops als ein weiterer Extremfall mit einem $\beta$ gleich 0 angesehen werden, bei der sich jeweils der Operator bzw. der MRO-Shop in der Wahl der Vertragsform durchsetzt. aus anderen Quellen kennt. Weiterhin kann man auf der Basis von Vertragsformen, die von den Verhandlungspartnern vorgeschlagen werden, auf die entsprechenden Präferenzen schließen und dieses Wissen in den Vertragsverhandlungen nutzen.

Die Wahl der Vertragsform wurde in diesem Aufsatz ausschließlich auf der Basis der Kosten- bzw. Risikostrukturen und der Sicherheitspräferenzen der Entscheidungsträger der beiden Vertragspartner erörtert. Auf die anderen Einflussfaktoren, die sich aus spezifischen Vor- und Nachteile der Vertragsformen ergeben, soll nicht eingegangen und auf die entsprechende Literatur verwiesen werden. ${ }^{18}$

\section{Zusammenfassung und Ausblick}

In diesem Aufsatz wurden die wichtigsten Vertragsformen der Maintenance ziviler Flugtriebwerke mit ihren Kostenund Risikostrukturen vorgestellt. Anhand dieser Strukturen und den Sicherheitspräferenzen der Entscheidungsträger wurden Rangfolgen der Vorteilhaftigkeit der Vertragsformen für den MRO-Shop und den Operator erarbeitet. Auf der Basis dieser Betrachtungen wurde weiterhin ein Modell formuliert, mit dem die fuir beide Vertragspartner optimale Vertragsform ausgewählt werden kann.

Dieses Themengebiet beinhaltet interessante Ansatzpunkte fuir weitergehende Untersuchungen. So besteht auf dem Gebiet des Wirtschaftsrechts der Bedarf, die Abbildung der Risikostrukturen in den Vertragswerken und die konkrete Umsetzung dieser Vertragsbestandteile in einem internationalen Umfeld intensiver zu analysieren. Im ingenieurwissenschaftlichen Bereich gilt es, die vielfältigen Möglichkeiten zur Reduzierung der Maintenance-Kosten (z. B. durch die Entwicklung neuer Reparaturverfahren) aufzudecken.

\begin{tabular}{|c|c|c|c|c|}
\hline \multirow{2}{*}{\multicolumn{2}{|c|}{$\beta=0,25$}} & \multicolumn{3}{|c|}{ Operator } \\
\hline & & Pessimist & Neutral & Optimist \\
\hline \multirow{3}{*}{ 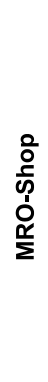 } & $\begin{array}{l}\frac{\tilde{W}}{E} \\
\frac{E}{W} \\
\mathscr{D} \\
0\end{array}$ & T\&M & $T \& M$ & $T \& M$ \\
\hline & $\begin{array}{l}\bar{\pi} \\
\overline{\frac{\pi}{5}} \\
\overline{0}\end{array}$ & $\mathrm{FbH}$ & $T \& M / F b h$ & $T \& M$ \\
\hline & 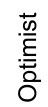 & $\mathrm{FbH}$ & $\mathrm{FbH}$ & $\mathrm{FbH}$ \\
\hline
\end{tabular}

\begin{tabular}{|c|c|c|c|c|}
\hline \multirow{2}{*}{\multicolumn{2}{|c|}{$\beta=0,5$}} & \multicolumn{3}{|c|}{ Operator } \\
\hline & & Pessimist & Neutral & Optimist \\
\hline \multirow{3}{*}{$\begin{array}{l}\frac{0}{0} \\
\frac{1}{\infty} \\
o ̛ \\
\frac{p}{\Sigma}\end{array}$} & 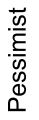 & GMC & $\mathrm{T} \& \mathrm{M} / \mathrm{GMC}$ & T\&M \\
\hline & 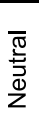 & $\mathrm{FbH}$ & GMC & T\&M \\
\hline & 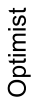 & $\mathrm{FbH}$ & $\mathrm{FbH}$ & T\&M \\
\hline
\end{tabular}

\begin{tabular}{|c|c|c|c|c|}
\hline \multirow{2}{*}{\multicolumn{2}{|c|}{$\beta=0,75$}} & \multicolumn{3}{|c|}{ Operator } \\
\hline & & Pessimist & Neutral & Optimist \\
\hline \multirow{3}{*}{$\begin{array}{l}\text { o } \\
\text { कर } \\
\text { ó } \\
\text { 官 }\end{array}$} & 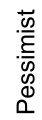 & $\mathrm{FbH}$ & GMC & GMC \\
\hline & 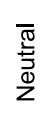 & $\mathrm{FbH}$ & GMC & GMC \\
\hline & 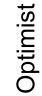 & $\mathrm{FbH}$ & GMC & GMC \\
\hline
\end{tabular}

Tab. 17: Optimale Vertragskompromisse bei unterschiedlicher Gewichtung des Einflusses der beiden Vertragspartner

Die in Tabelle 17 angegebenen optimalen Vertragskompromisse basieren auf dem in diesem Aufsatz betrachteten vereinfachten Beispiel bzw. dem gesetzten Einflussparameter $\beta$ und besitzen damit bis auf die beiden Extremfälle keinen grundsätzlich allgemeingültigen Charakter. Trotzdem können sie als Anhaltspunkt bei Vertragsverhandlungen dienen, wenn man z. B. die Präferenzen seines potenziellen Vertragspartners hinsichtlich unsicherer Ergebnisse aus früheren Verhandlungen oder 


\section{Anmerkungen}

1 Vgl. Rupp (2001), S. 1; Repp (2001), S. 3 ff.

2 Vgl. Duarte Leite (2003), S. 26 f.

3 MRO-Shop - Maintenance, Repair and Overhaul Shop

4 Auf weitere Faktoren der Steuerung geplanter Shop Visits wie z. B. Service Bulletins wird in diesem Aufsatz nicht eingegangen.

5 Hinsichtlich der Erfassung und Planung von Kosten sei auf die Kostenrechnungsliteratur verwiesen. Vgl. Kilger (1993), S. 231-677; Haberstock (1998), S. 55-103; Haberstock (1999), S. 107-256.

6 Vgl. Haberstock (1998), S. $67 \mathrm{ff}$.

7 Hinsichtlich der Zurechnung von aufgabenspezifisch relevanten Kosten Vgl. Kloock (1993), S. 193 ff. Mit einer derartigen beanspruchungsgerechten Zurechnung von Fixkosten werden den einzelnen Shop Visits Kapazitätskosten zugerechnet, deren Ausprägung unabhängig von der Beschäftigung des Shops und letztlich der Kapazitätsauslastung ist. In diesem Sinn kann die Kalkulationsfalle einer Vollkostenrechnung vermieden werden. Vgl. Dierkes (1993), S. 42 ff.; Kilger (1993), S. 857 f.; Dierkes (1998), S. 18.

8 Hinsichtlich der wichtigen, am Markt angebotenen Vertragsformen sei auf die Veröffentlichungen der einzelnen MRO-Shops auf den jeweiligen Homepages verwiesen. Vgl. z. B. www.geae.com; www.pwc.com; www.mtu.com 9 Vgl. Duarte Leite (2003), S. 28 f.

10 Grundsätzlich kann die Bewertung des Erfolges eines Vertrages anhand unterschiedlicher Ansätze erfolgen, die kompatibel zum grundsätzlichen Steuerungsmodell des entsprechenden Unternehmens gestaltet sein müssen. So könnte z. B. eine Bewertung auf Basis eines Kapitalwertes erfolgen, der sich aus der Summe der Barwerte der zukünftigen Cash Flows vor Einkommens-/Ertragsteuern und Finanzergebnis (CFBiT) ermitteln lässt. Auf weitere Erfolgsgrößen zur Steuerung von derartigen Verträgen wird aus Vereinfachungsgründen verzichtet.

11 Vgl. Duarte Leite (2003), S. 29.

12 Vgl. Duarte Leite (2003), S. 29.

13 Vgl. Rupp (2001), S. 2.

14 Vgl. zu den allgemeinen Ursachen von Abweichungen: Steglich (2001), S. $33 \mathrm{ff}$

15 Weitere Ursachen für Ergebnisabweichungen können in Wechselkursrisiken, inflationären Effekten auf dem Beschaffungsmarkt bzw. internen Störgrößen des Operators bzw. des MRO-Shops bestehen. Da für die beiden erstgenannten Effekte entsprechende Ansätze zur Gegensteuerung existieren und interne Effekte grundsätzlich von den beiden Vertragspartner beeinflussbar sind, sollen diese Abweichungsursachen in die Betrachtung der Risiko- bzw. Chancenstrukturen der Vertragsarten nicht mit einbezogen werden.

16 Vgl. Sieben/Schildbach (1994), S. 50 ff.

17 Vgl. Sieben/Schildbach (1994), S. 52 f.

18 Vgl. Duarte Leite (2003), S. 28 ff.

\section{Literatur}

Dierkes, S. (1998): Planung und Kontrolle von Prozeßkosten, Wiesbaden 1998.

Duarte Leite, J. (2003): Engine contract comparisons, in: The Engine Yearbook, Engine Yearbook 2003, S. 26-31.

Haberstock, L. (1998): Kostenrechnung I, 10. Aufl., bearbeitet durch V. Breithecker, Hamburg 1998.
Kilger, W. (1993): Flexible Plankostenrechnung und Deckungsbeitragsrechnung, 10. Aufl., bearbeitet durch K. Vikas, Wiesbaden 1993.

Kloock, J. (1993): Betriebliche Kostenpolitik aus planungsmäßiger und kalkulatorischer Sicht, in: R. Federmann (Hrsg.), Betriebswirtschaftslehre, Unternehmenspolitik und Unternehmensbesteuerung, Festschrift für G. Mann, Berlin 1993, S. 181-200.

Sieben, G./Schildbach, Th. (1994): Betriebswirtschaftliche Entscheidungstheorie, 4. Aufl., Düsseldorf 1994.

Steglich, M. (2001): Zielwertorientierte Auswertung von Kostenabweichungen, Wiesbaden 2001.

Repp, F. (2001): Assessment of the Different Costs Linked to Maintenance, in: Hüttl, T. et al. (Hrsg.), Tagungsband des DGLR Workshops „Maintenance von Flugzeugen und Triebwerken" am 5. und 6. Juli 2001, DGLR-Bericht 200103.

Rupp, O. (2001): Maintenance Cost for Civil Aircraft Engines, in: Hüttl, T. et al. (Hrsg.), Tagungsband des DGLR Workshops „Maintenance von Flugzeugen und Triebwerken“ am 5. und 6. Juli 2001, DGLR-Bericht 2001-03.

\section{Autor}

\section{Dr. Mike Steglich}

MTU Maintenance Berlin-Brandenburg GmbH

Dr.-Ernst-Zimmermann-Straße 2, 14974 Ludwigsfelde

Telefon +493378 824271

E-Mail: mike.steglich@lud.mtu.de 